\title{
Unconventional superconductivity mediated solely by isotropic electron-phonon interaction
}

\author{
Fabian Schrodi $\odot,{ }^{*}$ Peter M. Oppeneer $\odot,{ }^{\dagger}$ and Alex Aperis $\odot^{\ddagger}$ \\ Department of Physics and Astronomy, Uppsala University, P.O. Box 516, SE-75120 Uppsala, Sweden
}

(Received 23 June 2021; revised 7 September 2021; accepted 18 October 2021; published 28 October 2021)

\begin{abstract}
Unconventional superconductivity is commonly linked to electronic pairing mechanisms, since it is believed that the conventional electron-phonon interaction (EPI) cannot cause sign-changing superconducting gap symmetries. Here, we show that this common understanding needs to be revised when one considers a more elaborate theory of electron-phonon superconductivity beyond standard approximations. We self-consistently solve the full-bandwidth, anisotropic Eliashberg equations including vertex corrections beyond Migdal's approximation assuming the usual isotropic EPI for cuprate, Fe-based, and heavy-fermion superconductors with nested Fermi surfaces. In the case of the high- $T_{c}$ cuprates we find a $d$-wave order parameter, as well as a nematic state upon increased doping. For Fe-based superconductors, we obtain $s_{ \pm}$gap symmetry, while for heavy-fermion $\mathrm{CeCoIn}_{5}$ we find unconventional $d$-wave pairing. These results provide a proof of concept that EPI cannot be excluded as a mediator of unconventional and of high- $T_{c}$ superconductivity.
\end{abstract}

DOI: 10.1103/PhysRevB.104.L140506

Superconductors with unconventional-that is, non- $s$ wave-gap symmetries continue to attract great interest, because of their unique properties [1,2] and because they are closely linked to the as yet unexplained phenomenon of high- $T_{c}$ superconductivity $[3,4]$. Prominent material examples are the high- $T_{c}$ cuprates with $d$-wave and the iron-based superconductors with predominantly $s_{ \pm}$gap symmetry $[5,6]$. Members of these families share several common features such as, e.g., quasi-two-dimensional (2D) electronic band structures with good nesting properties, proximity to magnetic ordering, and strongly coupled superconductivity beyond the Bardeen-Cooper-Schrieffer (BCS) picture [7]. Resonances observed in inelastic neutron scattering experiments suggest the presence of spin fluctuations in the pairing mechanism $[5,8,9]$ but large isotope effects pointing at the involvement of phonons have also been measured [10-13]. These phonons have characteristic energies of a similar order of magnitude as those of the relevant spin fluctuations [14]. In this setting, the key for pinpointing the driving pairing mechanism has been the symmetry of the superconducting gap itself [5,9].

An unconventional gap is commonly considered as the signature of a repulsive, solely electronic mechanism that can be pairing if the electrons allow a sign change in their wave function. The most prominent example is the antiferromag-

\footnotetext{
*fabian.schrodi@physics.uu.se

†peter.oppeneer@physics.uu.se

${ }^{\ddagger}$ alex.aperis@physics.uu.se
}

Published by the American Physical Society under the terms of the Creative Commons Attribution 4.0 International license. Further distribution of this work must maintain attribution to the author(s) and the published article's title, journal citation, and DOI. Funded by Bibsam. netic spin-fluctuation mechanism where the wave vector of the dominant magnon excitations, which is associated with the nesting properties of the underlying electronic band structure of the material, matches the sign change of the superconducting gap [15]. Many superconductors comply with this picture, including cuprate, iron-based, and heavy-fermion materials [9].

Conversely, in the original theory of BCS [16] and its refinement by Eliashberg to include retardation effects [17], phonons mediate an effective attractive interaction between electrons which gives rise to a sign-preserving $s$-wave gap. Attempts to reconcile the phonon mechanism with unconventional gap symmetries do exist. For example, it has been demonstrated that a small- $q$-peaked electron-phonon interaction (EPI) can give rise to the unconventional gap of the cuprates and the Fe pnictides in the presence of enhanced Coulomb repulsion between electrons [18,19]. Despite recent observations of such a small- $q$ EPI in monolayer $\mathrm{FeSe} / \mathrm{SrTiO}_{3}$ [20], its existence in bulk superconductors is yet to be confirmed.

Notably, Eliashberg's theory for boson-mediated superconductivity rests on the premise of adiabaticity, i.e., the energy scale of electrons $\epsilon_{F}$ is much larger than that of the relevant bosons $\Omega$. In the case of phonons, Migdal showed that when the so-called nonadiabatic ratio $\alpha=\Omega / \epsilon_{F}$ is small $\left(\sim 10^{-2}\right)$, vertex corrections to the electron self-energy due to the EPI are negligible [21]. However, for many unconventional superconductors $\alpha \sim 0.1-0.3[7,22]$ and thus vertex corrections cannot be safely neglected. Including these requires solving the self-consistent Nambu-Dyson equation with crossing self-energy diagrams while retaining full frequency and momentum dependence, a task that has only recently been achieved [23]. Due to the numerical complexity of this problem, prior attempts adopted serious simplifications [24-27].

Here, we address the long-standing issue of phononmediated unconventional superconductivity by performing 
direct numerical solutions of the full-bandwidth anisotropic Eliashberg equations including the first vertex corrections to the self-energy beyond Migdal's approximation using material specific input for three archetypal unconventional superconducting systems, cuprates, iron pnictides, and heavyfermion $\mathrm{CeCoIn}_{5}$, that are known to exhibit quasi-twodimensional electronic structures as well as nested Fermi surfaces (FSs). In all our calculations, we assume an optical phonon mode that couples to electrons via a purely isotropic EPI which is the only interaction in the system.

We start with describing our model where electrons forming $n$ energy bands with dispersions $\xi_{\mathbf{k}, n}$ couple to Einstein phonons of frequency $\Omega$ via an isotropic and bandindependent EPI with coupling strength $g_{\mathbf{q}}=g_{0}$, where $\mathbf{q}=$ $\mathbf{k}-\mathbf{k}^{\prime}$. We consider the following Nambu form of the vertexcorrected electron self-energy for such a system,

$$
\hat{\Sigma}_{\mathbf{k}, m}=T \sum_{\mathbf{k}^{\prime}, n, m^{\prime}} g_{0}^{2} D_{m-m^{\prime}} \hat{\rho}_{3} \hat{G}_{\mathbf{k}^{\prime}, n, m^{\prime}} \hat{\rho}_{3} \hat{\Gamma}_{\mathbf{q}, m, m^{\prime}},
$$

with Matsubara frequencies $\omega_{m}=\pi T(2 m+1), m \in \mathbb{Z}$, at temperature $T$. For brevity we employ here the notation $f\left(\mathbf{k}, i \omega_{m}\right)=f_{\mathbf{k}, m}$ for any function $f$. The vertex renormalization function (hereafter denoted just the vertex function) is given by

$$
\hat{\Gamma}_{\mathbf{q}, m, m^{\prime}}=1+T \sum_{m^{\prime \prime}} g_{0}^{2} D_{m^{\prime}-m^{\prime \prime}} \hat{\Lambda}_{\mathbf{q}, m^{\prime \prime}, m^{\prime \prime}-m^{\prime}+m},
$$

with

$$
\hat{\Lambda}_{\mathbf{q}, m^{\prime \prime}, m^{\prime \prime}+l}=\sum_{\mathbf{k}^{\prime \prime}, n^{\prime}, n^{\prime \prime}} \hat{G}_{\mathbf{k}^{\prime \prime}, n^{\prime}, m^{\prime \prime}} \hat{\rho}_{3} \hat{G}_{\mathbf{k}^{\prime \prime}+\mathbf{q}, n^{\prime \prime}, m^{\prime \prime}+l} \hat{\rho}_{3}
$$

and $l=m-m^{\prime}$. Equation (1) includes an infinite series of Feynman diagrams that constitute the lowest-order Migdal self-energy and an infinite series of crossing diagrams that constitute the first vertex correction beyond Migdal's approximation $[23,25]$. Further details can be found in the Supplemental Material (SM) [28]. In the above, $D_{m-m^{\prime}}$ is the phonon propagator, while the electron Green's function in Nambu space with a Pauli basis $\hat{\rho}_{i}$ is given by

$$
\hat{G}_{\mathbf{k}, n, m}^{-1}=i \omega_{m} Z_{\mathbf{k}, m} \hat{\rho}_{0}-\phi_{\mathbf{k}, m} \hat{\rho}_{1}-\left[\xi_{\mathbf{k}, n}+\zeta_{\mathbf{k}, m}\right] \hat{\rho}_{3} .
$$

Here, $Z_{\mathbf{k}, m}, \zeta_{\mathbf{k}, m}$, and $\phi_{\mathbf{k}, m}$ describe, respectively, the mass and chemical potential renormalization, and the superconducting order parameter. From Eqs. (1)-(4) we derive a set of vertex-corrected, anisotropic, full-bandwidth, and multiband Eliashberg equations for these three functions, which are solved self-consistently without further approximation [23,28,29]. From the results we obtain the gap function via $\Delta_{\mathbf{k}, m}=\phi_{\mathbf{k}, m} / Z_{\mathbf{k}, m}$, and the gap edge $\Delta(\mathbf{k}) \approx \Delta_{\mathbf{k}, m=0}$.

We model the electron band structure of cuprate superconductors with the commonly employed tight-binding model, $\xi_{\mathbf{k}}=\beta_{\mathbf{k}}-\delta_{\mathbf{k}}-\mu$, that includes nearest- and next-nearestneighbor hopping terms $\beta_{\mathbf{k}}=t\left[\cos k_{x}+\cos k_{y}\right]$ and $\delta_{\mathbf{k}}=C-$ $t^{\prime} \cos k_{x} \cos k_{y}$, respectively. Unless noted otherwise we set the hopping energies $t, t^{\prime}$, parameter $C$, and chemical potential $\mu$ to $\left(C, t, t^{\prime}, \mu\right)=(0,-0.25,0.1,-0.07) \mathrm{eV}$. We further adopt $g_{0}=148 \mathrm{meV}$ and choose a phonon frequency $\Omega=$ $50 \mathrm{meV}$ that is motivated from angle-resolved photoemission spectroscopy (ARPES) measurements on different cuprate systems [14]. For Fe-based materials the scattering strength and characteristic phonon frequency are chosen as $g_{0}=$ $130 \mathrm{meV}$ and $\Omega=17 \mathrm{meV}$ [30-32]. The respective electronic energies are described by a two-band model with $\xi_{\mathbf{k}}^{( \pm)}=\beta_{\mathbf{k}} \pm$ $\delta_{\mathbf{k}}-\mu$ and parameters $\left(C, t, t^{\prime}, \mu\right)=(1 / 3,1 / 6,1 / 12,0) \mathrm{eV}$. In the case of $\mathrm{CeCoIn}_{5}$ we use the two-band tight-binding model of Ref. [33] (which neglects several small FS pockets) and choose $\Omega=5 \mathrm{meV}$ [34] and $g_{0}=4 \mathrm{meV}$. Qualitatively similar results as presented here are also found for larger frequencies and different $g_{0}$ values (see SM [28]).

The resulting Fermi surfaces for our cuprate, Fe-based, and

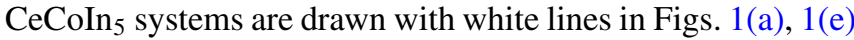
and 1(i), respectively. The corresponding calculated noninteracting charge susceptibilities,

$$
\mathcal{X}_{\mathbf{q}, 0}^{(0)}=\sum_{\mathbf{k}, n, n^{\prime}} \frac{n_{F}\left(\xi_{\mathbf{k}, n}\right)-n_{F}\left(\xi_{\mathbf{k}+\mathbf{q}, n^{\prime}}\right)}{\xi_{\mathbf{k}, n}-\xi_{\mathbf{k}+\mathbf{q}, n^{\prime}}}
$$

with Fermi-Dirac functions $n_{F}(\cdot)$, are shown in Figs. 1(d), 1(h) and 1(1). As expected, these exhibit pronounced peaks at wave vectors $\mathbf{Q}=(\pi, \pi)$ for cuprate and Fe-based systems, and $\mathbf{Q} \approx(\pi / 2, \pi / 2)$ for $\mathrm{CeCoIn}_{5}$. These pronounced peaks evidence directly the well-known good nesting properties of these three quasi-2D systems. Note that for $\mathrm{CeCoIn}_{5}$ there are several FS parts involved which causes a broadening of the $(\pi / 2, \pi / 2)$ peaks. In spin-fluctuation theories for these systems, such susceptibilities provide the necessary momentum-dependent repulsive interaction that gives rise to unconventional $\Delta(\mathbf{k})$ symmetries (see Ref. [9]). Here, we solve numerically the Eliashberg equations derived from Eqs. (1)-(3) to determine the gap symmetry.

The main results of this Letter are shown in Figs. 1(a), 1(e) and 1(i) where we plot our self-consistently calculated momentum dependence of the superconducting gap for each of the three systems that we consider. Remarkably, as shown in Fig. 1(a), for the cuprate case we find a clear $d_{x^{2}-y^{2}}$ gap with a Brillouin-zone (BZ) modulation that is roughly proportional to $\cos k_{x}-\cos k_{y}$ and a realistic amplitude $\max _{\mathbf{k}} \Delta(\mathbf{k}) \simeq 5.4 \mathrm{meV}$ at $T=30 \mathrm{~K}$. We note that previously it was found that a $d_{x^{2}-y^{2}}$ symmetry is possible for a pure phononic Holstein model using a cluster dynamical mean-field theory approximation [35]. For the Fe-based system, shown in Fig. 1(e), we find the $s_{ \pm}$unconventional gap with $\Delta(\mathbf{k}) \propto \cos k_{x}+\cos k_{y}$ and a realistic amplitude $\max _{\mathbf{k}} \Delta(\mathbf{k}) \simeq 4.2 \mathrm{meV}$ at $T=15 \mathrm{~K}$. Lastly, for CeCoIn 5 , shown in Fig. 1(i), we find a higher-harmonic $d_{x^{2}-y^{2}}$-gap symmetry with $\Delta(\mathbf{k}) \propto \cos 2 k_{x}-\cos 2 k_{y}$ and $\max _{\mathbf{k}} \Delta(\mathbf{k}) \simeq$ $0.87 \mathrm{meV}$ at $T=2 \mathrm{~K}$ which is also in reasonable agreement with experiment [36].

Our calculations reveal that the strongly momentumdependent gap symmetries in these superconductors can be obtained solely via isotropic EPI provided that vertex corrections to the electron self-energy are taken into account. Without vertex corrections, we obtain $s$-wave symmetry, commonly associated with isotropic EPI. A crucial ingredient for this result is the fact that the vertex correction in Eq. (2) is inherently momentum dependent regardless of the bare EPI being momentum independent. To better understand this point, we calculate the static vertex by plugging our selfconsistent results back into Eq. (2) and taking $m=m^{\prime}=0$. 


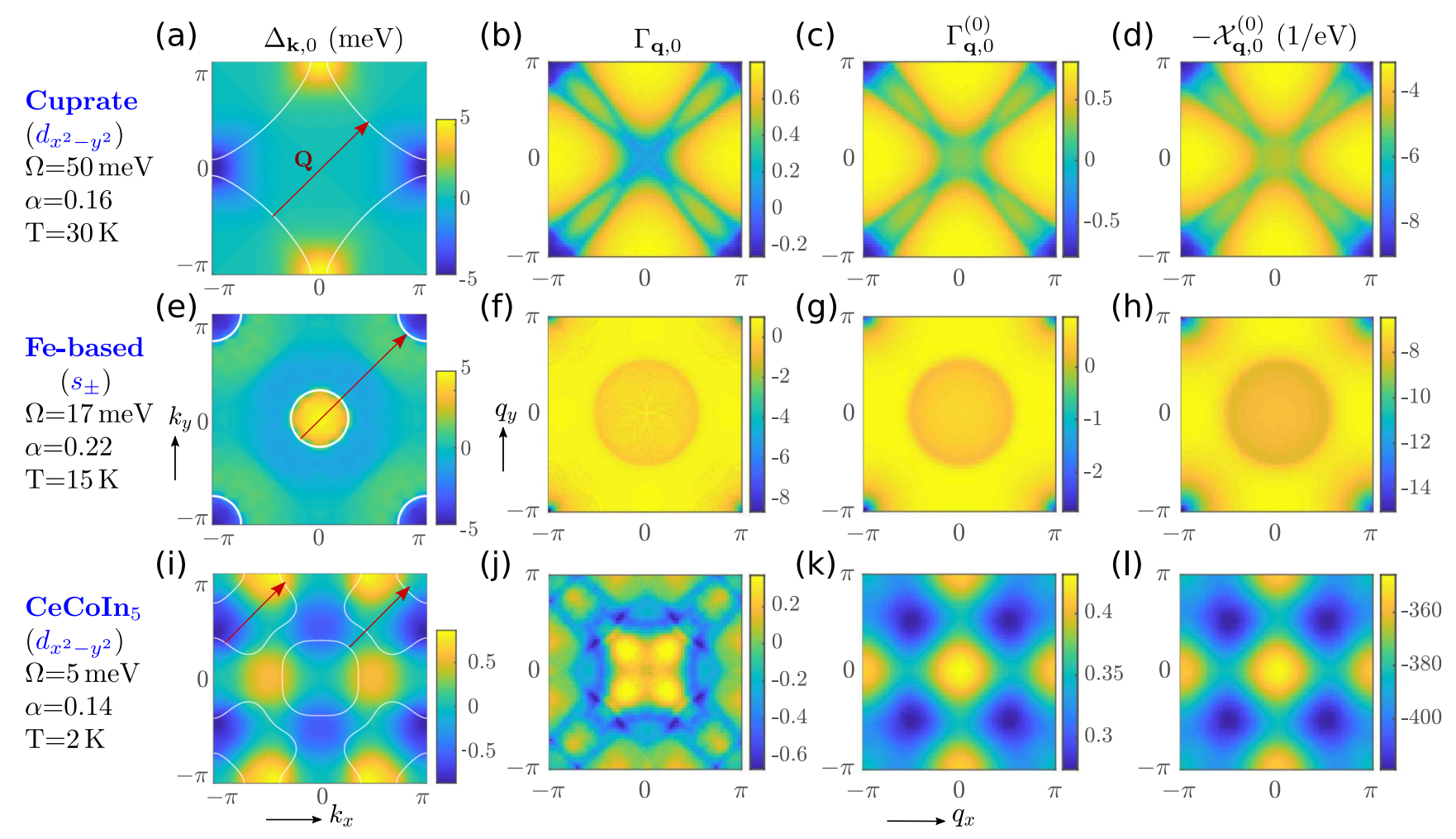

FIG. 1. Self-consistently calculated unconventional superconductivity. (a), (e), (i) The unconventional superconducting gap calculated by direct solution of the vertex-corrected Eliashberg equations. The Fermi surface is in each case drawn with white lines in the Brillouin zone. Fermi-surface nesting is indicated by the red vector $\mathbf{Q}$. (b), (f), (j) The self-consistently calculated vertex function, and (c), (g), (k), the calculated bare vertex function for $T>T_{c}$. (d), (h), (l) The calculated bare charge susceptibility. The first row corresponds to results for cuprates, where a $d_{x^{2}-y^{2}}$ gap symmetry is obtained. The second row corresponds to Fe-based materials where an $s_{ \pm}$symmetry is found. The third row corresponds to $\mathrm{CeCoIn}$ where we obtain a higher-harmonic $d_{x^{2}-y^{2}}$ gap symmetry. The used phonon frequency $\Omega$, nonadiabaticity ratio $\alpha$, and temperature $T$ are given in the legend.

In the static case all channels share the same vertex which is then a scalar given by

$$
\Gamma_{\mathbf{q}, 0}=1+g_{0}^{2} T \sum_{m^{\prime \prime}} D_{m^{\prime \prime}} \frac{1}{2} \operatorname{Tr}\left\{\hat{\Lambda}_{\mathbf{q}, \mathrm{m}^{\prime \prime}}\right\} .
$$

The calculated static vertices, shown in Figs. 1(b), 1(f) and 1(j), have momentum structures that share similar characteristics. $\Gamma_{\mathbf{q}, 0}$ is strongly peaked and negative, i.e., repulsive, for wave vectors that almost or perfectly coincide with the nesting wave vectors that correspond to the susceptibility peaks of Figs. 1(d), 1(h) and 1(l) and positive otherwise. These large repulsive peaks allow pairing with a sign change across the BZ, thus providing a mechanism for unconventional superconductivity in a manner similar to spin fluctuations. However, in contrast to the latter, where the small wave-vector part of the repulsive interaction can interfere destructively to pairing [37-39], in our case the EPI remains attractive at smaller wave vectors and therefore can contribute to the unconventional pairing. We therefore conclude that the vertex-corrected EPI in all three prototypical examples is selfconsistently optimized so as to maximize the pairing. When we start the procedure with an initial $s$-wave order parameter, it changes during the self-consistent calculations to the here-presented unconventional order parameters. Mathemat- ically, this is possible because the self-consistent procedure corresponds to minimizing the system's free energy, and the superconducting gap and vertex functions are allowed to mutually influence each other during the self-consistent cycle. Physically, this can be understood as the result of the formed Cooper pairs dynamically renormalizing the EPI and vice versa.

To take our analysis further, we draw a much more simplified picture where we make a one-loop approximation to Eq. (6) by using noninteracting Green's functions, $\hat{G}_{\mathbf{k}, n, m}^{(0)}=$ $\left[i \omega_{m}-\xi_{\mathbf{k}, n} \hat{\rho}_{3}\right]^{-1}$. The resulting bare static vertices $\Gamma_{\mathbf{q}, 0}^{(0)} \mathrm{calcu}-$ lated for our three cases are shown in Figs. 1(c), 1(g) and 1(k). For cuprate and Fe-based systems, these are roughly similar to their respective interacting vertices [see Figs. 1(b) and 1(f)]. However, for $\mathrm{CeCoIn}_{5}$, that has a more complex FS, the bare vertex shown in Fig. 1(k) is positive everywhere in the BZ in contrast to the respective self-consistent interacting vertex of Fig. 1(j). This case presents a clear example where inclusion of the back-reaction of the superconducting gap to the vertex function is essential for finding the correct gap symmetry and therefore for the occurrence of unconventional pairing. This also implies that although the much-easier-to-calculate bare vertex function may serve as an indicator of possible unconventional superconductivity due to the vertex-corrected EPI, 

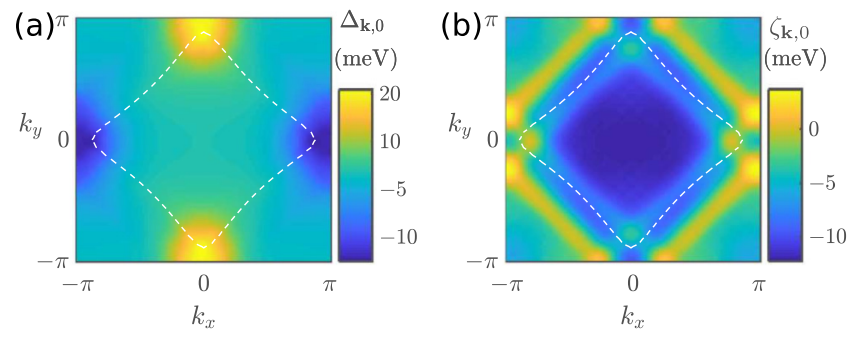

FIG. 2. Self-consistently calculated nematic superconductivity. (a) The calculated nematic superconducting gap $\Delta_{\mathbf{k}, 0}$ for a cuprate Fermi surface (white dashed line) in the slightly hole-doped regime, $\left(C, t, t^{\prime}, \mu\right)=(0,-1 / 4,1 / 10,-0.11) \mathrm{eV}$. (b) The computed zeroenergy chemical potential renormalization, $\zeta_{\mathbf{k}, m=0}$.

it may nevertheless miss cases with more complex FSs, and therefore, full self-consistent calculations are indispensable for accurate predictions.

As can be seen in Fig. 1, apart from possible different signs, the bare vertices are very similar to the respective bare susceptibilities for each system. The results shown in Figs. 1(d), 1(h) and 1 (l) correspond to the noninteracting case $\mathcal{X}_{\mathbf{q}, 0}^{(0)}$. One can observe that the second term on the right-hand side of Eq. (6) is proportional to the charge susceptibility weightened by the phonon propagator $D_{m}$. Since in our simple Einstein phonon picture this weightening takes place only in frequency space, it is responsible for making parts of the EPI repulsive and parts of it attractive, while the overall momentum structure follows that of the charge susceptibility.

To strengthen this argument further, we have also computed the renormalized charge susceptibilities below $T_{c}$. These can be calculated from Eq. (3) as $\mathcal{X}_{\mathbf{q}, 0}=$ $(1 / 2) T \sum_{m^{\prime \prime}} \operatorname{Tr}\left\{\hat{\Lambda}_{\mathbf{q}, \mathrm{m}^{\prime \prime}}\right\}$ and are given in the SM [28]. They are very similar to the interacting vertex functions shown in Fig. 1. Importantly, the renormalized charge susceptibilities $\mathcal{X}_{\mathbf{q}, 0}$ do not diverge and therefore an instability to a charge density wave is not expected here.

Another interesting outcome of our theory for the cuprates is the possibility to obtain self-consistently nematic superconductivity. The so-called nematic state, in which $C_{4}$ rotational symmetry is reduced to a $C_{2}$ orthorhombic structure, occupies a well-established region in the temperature-doping phase diagrams of cuprate superconductors [40]. For convenience of the calculations, we here use $g_{0}=170 \mathrm{meV}$, giving $T_{c} \approx$ $140 \mathrm{~K}$ [41]. We also shift the dispersion towards hole doping by setting $\mu=-0.11 \mathrm{eV}$. The resulting FS is drawn in Fig. 2 (white-dashed lines). The rest of the parameters are unchanged. Solving the vertex-corrected Eliashberg equations we observe a spontaneous change from $C_{4}$ to $C_{2}$ BZ symmetry in all three functions $\Delta_{\mathbf{k}, m=0}, \zeta_{\mathbf{k}, m=0}$, and $Z_{\mathbf{k}, m=0}$. The first two are shown in Figs. 2(a) and 2(b), respectively.

The superconducting gap of Fig. 2(a) is an admixture of $d_{x^{2}-y^{2}}+s$ symmetries that lowers $C_{4}$ symmetry to $C_{2}$ and is therefore nematic. Inspection of the chemical potential renormalization in Fig. 2(b) similarly reveals a twofold symmetric energy band renormalization which we can identify as an induced Pomeranchuk order parameter [42]. Nematicity appears only at low energies, so we always obtain anisotropic $s$-wave symmetry solutions for $m \gg 1$. It disappears for
$T>T_{c}$ where we find a restored $C_{4}$ symmetry in $\zeta_{\mathbf{k}, 0}$ and $Z_{\mathbf{k}, 0}$. These results indicate that the vertex-corrected renormalized EPI may very well be simultaneously pairing even in seemingly competing pairing channels, such as those of $s$-wave and higher angular momentum, which, depending on the underlying band structure, may lead to nematic superconductivity being the energetically favored solution.

Apart from the unconventional gap symmetry, another frequent argument against the relevance of the EPI in unconventional high- $T_{c}$ superconductors is a seemingly small coupling constant [43]. However, full-bandwidth Eliashberg theory generally includes Cooper pairing of states away from the Fermi level which may contribute to the gap size and $T_{c}$ [44]. A similar behavior can be expected in vertexcorrected full-bandwidth theory [23]. Focusing on the cuprate case, we carried out the computationally heavy task of solving for the complete self-consistent temperature dependence of our vertex-corrected Eliashberg equations. Within our chosen parameter set, we find a realistic $T_{c} \approx 52 \mathrm{~K}$. We furthermore estimated that for $T>T_{c}$, the effective electronphonon coupling is $\lambda_{m} \approx 0.34$ (see SM [28]). Therefore, seemingly weak-coupling values are nonetheless compatible with phonon-mediated high- $T_{c}$ unconventional superconductivity.

We note that our findings do not exclude that spin fluctuations play a role. They can, e.g., be important for the formation of a quantum critical point [9]. They can also contribute to Cooper pairing, since both EPI and spin fluctuations can lead to a pairing symmetry derived from the FS nesting, and hence, both mechanisms can act cooperatively. To establish unambiguously the relative size of their contributions, selfconsistent simulations within multichannel vertex-corrected Eliashberg theory will be required to treat both bosonic mediators on equal footing. We further comment that, with $\alpha \approx$ $0.1-0.2$, we are in the weakly nonadiabatic regime. Therefore, we do not expect that higher-order vertex corrections will influence our result much. Lastly, we mention that we have neglected the influence of the Coulomb repulsion, which does not affect our results, as the direct electron-electron Coulomb repulsion cancels out for an unconventional order parameter.

Our vertex-corrected Eliashberg-theory calculations provide a proof of principle that isotropic EPI can give rise to unconventional superconductivity. This phonon-mediated unconventional superconductivity relies on Fermi-surface nesting and moderate nonadiabaticity that are present in many unconventional superconductors $[7,9]$. The latter gives rise to a repulsive EPI which is antipairing in the $s$-wave channel [23] while the former makes this interaction peak near the nesting wave vector(s) and thus favorable for unconventional pairing in a similar fashion as purely electronic mechanisms such as spin fluctuations. Our results show that the conventional EPI can mediate unconventional superconductivity and highlight the possibility that electron-phonon coupling plays a substantial role in any superconductor with unconventional gap symmetry including the high- $T_{C}$ s.

This work has been supported by the Swedish Research Council (VR), the Röntgen-Angström Cluster, and the Knut and Alice Wallenberg Foundation (Grant No. 2015.0060). 
The calculations were enabled by resources provided by the Swedish National Infrastructure for Computing (SNIC) at
NSC Linköping, partially funded by the Swedish Research Council through Grant Agreement No. 2018-05973.
[1] M. Sigrist and K. Ueda, Rev. Mod. Phys. 63, 239 (1991).

[2] X.-L. Qi and S.-C. Zhang, Rev. Mod. Phys. 83, 1057 (2011).

[3] 100 Years of Superconductivity, edited by H. Rogalla and P. H. Kes (CRC Press, Boca Raton, FL, 2011).

[4] B. Keimer, S. A. Kivelson, M. R. Norman, S. Uchida, and J. Zaanen, Nature (London) 518, 179 (2015).

[5] C. C. Tsuei and J. R. Kirtley, Rev. Mod. Phys. 72, 969 (2000).

[6] G. R. Stewart, Rev. Mod. Phys. 83, 1589 (2011).

[7] Y. J. Uemura, L. P. Le, G. M. Luke, B. J. Sternlieb, W. D. Wu, J. H. Brewer, T. M. Riseman, C. L. Seaman, M. B. Maple, M. Ishikawa, D. G. Hinks, J. D. Jorgensen, G. Saito, and H. Yamochi, Phys. Rev. Lett. 66, 2665 (1991).

[8] P. Dai, Rev. Mod. Phys. 87, 855 (2015).

[9] D. J. Scalapino, Rev. Mod. Phys. 84, 1383 (2012).

[10] G.-H. Gweon, T. Sasagawa, S. Y. Zhou, J. Graf, H. Takagi, D.H. Lee, and A. Lanzara, Nature (London) 430, 187 (2004).

[11] H. Iwasawa, J. F. Douglas, K. Sato, T. Masui, Y. Yoshida, Z. Sun, H. Eisaki, H. Bando, A. Ino, M. Arita, K. Shimada, H. Namatame, M. Taniguchi, S. Tajima, S. Uchida, T. Saitoh, D. S. Dessau, and Y. Aiura, Phys. Rev. Lett. 101, 157005 (2008).

[12] R. H. Liu, T. Wu, G. Wu, H. Chen, X. F. Wang, Y. L. Xie, J. J. Ying, Y. J. Yan, Q. J. Li, B. C. Shi, W. S. Chu, Z. Y. Wu, and X. H. Chen, Nature (London) 459, 64 (2009).

[13] R. Khasanov, M. Bendele, A. Bussmann-Holder, and H. Keller, Phys. Rev. B 82, 212505 (2010).

[14] A. Lanzara, P. V. Bogdanov, X. J. Zhou, S. A. Kellar, D. L. Feng, E. D. Lu, T. Yoshida, H. Eisaki, A. Fujimori, K. Kishio, J.-I. Shimoyama, T. Noda, S. Uchida, Z. Hussain, and Z.-X. Shen, Nature (London) 412, 510 (2001).

[15] D. J. Scalapino, E. Loh, and J. E. Hirsch, Phys. Rev. B 34, 8190 (1986).

[16] J. Bardeen, L. N. Cooper, and J. R. Schrieffer, Phys. Rev. 108, 1175 (1957).

[17] G. M. Eliashberg, Sov. Phys. JETP 11, 696 (1960).

[18] G. Varelogiannis, Phys. Rev. B 57, 13743 (1998).

[19] A. Aperis, P. Kotetes, G. Varelogiannis, and P. M. Oppeneer, Phys. Rev. B 83, 092505 (2011).

[20] J. J. Lee, F. T. Schmitt, R. G. Moore, S. Johnston, Y.-T. Cui, W. Li, M. Yi, Z. K. Liu, M. Hashimoto, Y. Zhang, D. H. Lu, T. P. Devereaux, D.-H. Lee, and Z.-X. Shen, Nature (London) 515, 245 (2014).

[21] A. B. Migdal, Sov. Phys. JETP 34, 996 (1958).

[22] P. Benedetti, C. Grimaldi, L. Pietronero, and G. Varelogiannis, Europhys. Lett. 28, 351 (1994).

[23] F. Schrodi, P. M. Oppeneer, and A. Aperis, Phys. Rev. B 102, 024503 (2020).

[24] C. Grimaldi, L. Pietronero, and S. Strässler, Phys. Rev. Lett. 75, 1158 (1995).

[25] M. Botti, E. Cappelluti, C. Grimaldi, and L. Pietronero, Phys. Rev. B 66, 054532 (2002).

[26] P. Miller, J. K. Freericks, and E. J. Nicol, Phys. Rev. B 58, 14498 (1998).

[27] J. P. Hague, J. Phys.: Condens. Matter 15, 2535 (2003).

[28] See Supplemental Material at http://link.aps.org/supplemental/ 10.1103/PhysRevB.104.L140506 for the complete formalism of vertex-corrected Eliashberg theory, details on the tightbinding models used for the cuprate, Fe-based, and heavyfermion superconductors, and explicit convergence studies for the momentum and Matsubara frequency grids. Furthermore, additional results are presented for the superconducting gaps varying the EPI interaction strength, phonon frequencies, and temperatures, as well as full calculations of the temperature dependence of the cuprate superconducting gap. Also, the calculated renormalized charge susceptibilities and results for the electron-phonon coupling constant are given.

[29] The Uppsala Superconductivity (UppSC) code provides a package to self-consistently solve the anisotropic, multiband, and full-bandwidth Eliashberg equations for frequency-even and frequency-odd superconductivity mediated by phonons, and charge or spin fluctuations on the basis of ab initio calculated input.

[30] A. A. Kordyuk, V. B. Zabolotnyy, D. V. Evtushinsky, T. K. Kim, I. V. Morozov, M. L. Kulić, R. Follath, G. Behr, B. Büchner, and S. V. Borisenko, Phys. Rev. B 83, 134513 (2011).

[31] M. Zbiri, H. Schober, M. R. Johnson, S. Rols, R. Mittal, Y. $\mathrm{Su}, \mathrm{M}$. Rotter, and D. Johrendt, Phys. Rev. B 79, 064511 (2009).

[32] L. Boeri, O. V. Dolgov, and A. A. Golubov, Phys. C: Superconductivity 469, 628 (2009).

[33] J. S. Van Dyke, F. Massee, M. P. Allan, J. C. S. Davis, C. Petrovic, and D. K. Morr, Proc. Natl. Acad. Sci. USA 111, 11663 (2014).

[34] H. Martinho, P. G. Pagliuso, V. Fritsch, N. O. Moreno, J. L. Sarrao, and C. Rettori, Phys. Rev. B 75, 045108 (2007).

[35] J. P. Hague, Phys. Rev. B 73, 060503(R) (2006).

[36] Y. Fasano, P. Szabó, J. Kacmarcik, Z. Pribulova, P. Pedrazzini, P. Samuely, and V. F. Correa, Phys. B: Condens. Matter 536, 798 (2018).

[37] F. Schrodi, A. Aperis, and P. M. Oppeneer, Phys. Rev. B 102, 014502 (2020).

[38] H. Yamase and T. Agatsuma, Phys. Rev. B 102, 060504(R) (2020).

[39] F. Schrodi, A. Aperis, and P. M. Oppeneer, Phys. Rev. B 102, 180501(R) (2020).

[40] E. Fradkin, S. A. Kivelson, M. J. Lawler, J. P. Eisenstein, and A. P. Mackenzie, Annu. Rev. Condens. Matter Phys. 1, 153 (2010).

[41] Using a larger $g_{0}$ leads to a higher $T_{c}$, therefore allowing us to work at higher temperatures that demand fewer Matsubara frequencies and are thus less computationally demanding. For $g_{0}=170 \mathrm{meV}$ and the same parameter set as used in Fig. 1(a) we find the same $d$-wave symmetry but with a larger gap value and $T_{c} \approx 140 \mathrm{~K}$.

[42] G. Livanas, A. Aperis, P. Kotetes, and G. Varelogiannis, Phys. Rev. B 91, 104502 (2015).

[43] F. Giustino, M. L. Cohen, and S. G. Louie, Nature (London) 452, 975 (2008).

[44] A. Aperis and P. M. Oppeneer, Phys. Rev. B 97, 060501(R) (2018). 